\title{
Nano Topological Analysis For Power System Control
}

\author{
M.Kamel EL-Sayed \\ Department of Basic Science \\ Higher Institute of Engineering and Technology, Kafr El-Sheikh, Egypt. \\ dr.mkamel123@gmail.com
}

\section{ABSTRACT}

In this paper,we introduce an approach for analysis of information concerning electrical power system. The suggested method is a result of hybridizing rough set concepts with nano topology constructed on the set of all data using the boundary of uncertain decision sets and its lower approximation. Bases of nano topologies are used as indicators for selecting effective features in information system of a power control. This method is applied using the main experimental data which make the suggested model near from the real life information.

\section{Keywords}

Rough Sets, Nano Topology, Lower Approximation, Upper Approximation, Core.

\section{INTRODUCTION}

Electrical power system is one of the most complex artificial systems in this world, which safe, steady, economical and reliable operation plays a very important part in social economic development, even in social stability due to high degree of uncertainty and the large number of variables involved. The various supervision and control actions require the presence of an operator, who must be capable of efficiently responding to the most diverse requests, by handling various types of data and information $[3,4,16]$.

Rough set theory (RST) is a relatively new mathematical and Al technique introduced in the early 1980's by Pawlak $[8,9,10,11,12]$ The technique is particularly suited to reasoning about imprecise or incomplete data, and discovering relationships in this data. A classification is simply a partition of a universe[14]. Thus, in rough set theory, objects are partitioned into equivalence classes based on their attribute-values, which are essentially functional information associated with the objects. Many databases only contain functional information about objects; however, data about the relationships between objects has become increasingly important in decision analysis.

The main advantage of rough set theory is that it does not require any preliminary or additional information about data- like probability in statistics, basic probability assignment in Dempster Shafer theory of evidence or the value of possibility in fuzzy set theory. Lellis Thivagar et al [15] introduced a nano topological space with respect to a subset $X$ of an universe which is defined in terms of lower and upper approximations of X. The elements of a nano topological space are called the nano-open sets. He has also studied nano closure and nano interior of a set.

The word "nano" itself refers to the length scale (one nanometre is one billionth of a metre) that is one thousand times smaller than the micro scale, the scale that was traditionally associated with the electronics industry. Viruses and DNA are examples of natural objects on the nano scale, in contrast a human cell can appear enormous. The term nanotechnology refers to the engineering, measurement understanding of nano-scaled materials and devices.

The elements of nano topology can be characterized by through two crisp sets, called lower and upper approximations belong to the set. Following these definitions, the boundary region is the difference between the two approximation and we have applied the concept of topological basis to find the deciding factors in power system control.

In this paper, we apply nano topology to find the deciding factors in power system control.

\section{1- Preliminaries}

Definition 1.1 [2,4,7,13]: Let $\boldsymbol{U}$ be a non-empty finite set of objects called the universe and $\boldsymbol{R}$ be an equivalence relation on $\boldsymbol{U}$ named as the indiscernibility relation. The pair $(\boldsymbol{U}, \boldsymbol{R})$ is called the approximation space. Let $\boldsymbol{X}$ be a subset of $\boldsymbol{U}$.

i) The lower approximation of $\boldsymbol{X}$ with respect to $\boldsymbol{R}$ is the is the set of all objects, which can be for certain classified as $\boldsymbol{X}$ with respect to $\boldsymbol{R}$ and it is denoted by $\boldsymbol{R}_{*}(\boldsymbol{X})$

$$
\boldsymbol{R}_{*}(X)=\bigcup_{x \in u}\{R(x): R(X) \subseteq X\} \text { where } R(x)
$$

denotes the equivalence class determined by $X$

ii) The upper approximation of $\boldsymbol{X}$ with respect to $\boldsymbol{R}$ is the set of all objects, which can be possibly classified as $\boldsymbol{X}$ with respect to $\boldsymbol{R}$ and it is denoted by $\boldsymbol{R}^{*}(\boldsymbol{X})$ That is, 


$$
\boldsymbol{R}^{*}(\boldsymbol{X})=\bigcup_{x \in u}\{\boldsymbol{R}(\boldsymbol{x}): \boldsymbol{R}(\boldsymbol{X}) \cap \boldsymbol{X} \neq \boldsymbol{\phi}\}
$$

iii) The boundary region of $\boldsymbol{X}$ with respect to $\boldsymbol{R}$ is the set of all objects, which can be classified neither as $\boldsymbol{X}$ nor as not- $\boldsymbol{X}$ with respect to $\boldsymbol{R}$ and it is denoted by $\boldsymbol{B}_{\boldsymbol{R}}(\boldsymbol{X})$. That is, $\boldsymbol{B}_{\boldsymbol{R}}(\boldsymbol{X})=\boldsymbol{R}^{*}(\boldsymbol{X})-\boldsymbol{R}_{*}(\boldsymbol{X})$

The set $X$ is said to be rough with respect to $R$ if $R^{*}(X) \neq R^{*}(X)$. That is, if $B R(X) \neq \varphi$.

\section{Proposition 1.2 [6]:}

If $(\boldsymbol{U}, \boldsymbol{R})$ is an approximation space and $\boldsymbol{X}$ and $\boldsymbol{Y}$ are subsets of $\boldsymbol{U}$, then
i) $R^{\star}(X) \subseteq X \subseteq R^{*}(X)$
ii) $R^{*}(\varphi)=R^{*}(\varphi)=\varphi$ and $R^{*}(U)=R^{*}(U)=U$
iii) $R^{*}(X \cup Y)=R^{*}(X) \cup R^{*}(X)$
iv) $R_{*}(X \cup Y) \supseteq R_{*}(X) \cup R^{*}(Y)$
v) $R_{*}(X \cap Y)=R_{*}(X) \cap R_{*}(Y)$
vi) $R^{*}(X \cap Y) \subseteq R^{*}(X) \cap R^{*}(Y)$
vii) $R^{*}(X) \subseteq R^{*}(Y)$ and $R^{*}(X) \subseteq R^{*}(Y)$ whenever $X \subseteq Y$
viii ) $R^{*}(X C)=\left[R^{*}(X)\right] C$ and $R^{*}(X C)=\left[R^{*}(X)\right] C$
ix) $R * R *(X)=R^{*} R *(X)=R *(X)$
x) $R^{\star} R^{*}(X)=R * R^{*}(X)=R^{*}(X)$

Remark 1.3: $R^{*}: P(U) \rightarrow P(U)$ satisfies the Kuratowski closure axioms that
i) $R^{*}(\varphi)=\varphi$
ii) $X \subseteq R^{*}(X)$
iii) $R^{*}(X \cup Y)=R^{*}(X) \cup R^{*}(X)$
iv) $R^{*} R^{*}(X)=R^{*}(X)$ for all subsets $X$ and $Y$ of $U$

If $F=\left\{X \subseteq U / R^{*}(X)=X\right\}$, using conditions (i) to (iv), we see that $\varphi$ and

$U$ are in $F$;

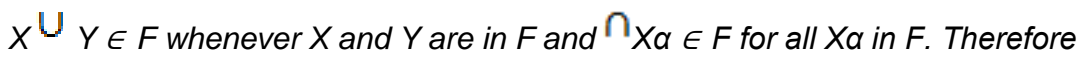

the family $T$, of complements of members of $F$ is a topology on $U$. Thus, $F$ is

the family of T-closed sets. Also, $\mathrm{Cl}(X)=R^{*}(X)$. Therefore, $R^{*}$ is the

Kuratowski's closure operatorRemark 2.4: Since $R^{*}: P(U) \rightarrow P(U)$ satisfies the

following properties that
v) $R *(U)=U$
vi) $R *(X) \subseteq X$
vii) $R_{*}^{*}(X \cap Y)=R^{*}(X) \cap R^{*}(Y)$
viii) $R * R^{*}(X)=R^{*}(X)$ for all subsets $X$ and $Y$ of $U$, the operator $R^{*}$ is the Interior operator.

\section{2- Nano Topology}

In this section we use nano topology Lellis Thivagar et al [15] in terms of the lower and upper approximations.

Remark 2.1: Let $U$ be the universe of objects and $R$ be an equivalence relation on $U$. For $X \subseteq U$, we define

$\tau_{R}=\left\{U, \varphi, R^{*}(X), R^{*}(X), B_{R}(X)\right\}$, where $R^{*}(X), R^{*}(X)$ and $B_{R}(X)$ are respectively the upper approximation, the lower approximation and the boundary region of $X$ with respect to $R$. We note that $U$ and $\varphi \in \tau_{R}$.

Since $R^{*}(X) \subseteq R^{*}(X), R^{*}(X) \cup R^{*}(X)=R^{*}(X) \in \tau_{R^{*}}$ Also, 
$R^{*}(X) \cup B_{R}(X)=R^{*}(X) \in \boldsymbol{\tau}_{\boldsymbol{R}}$ and $R^{*}(X) \cup B_{R}(X)=R^{*}(X) \in \boldsymbol{\tau}_{\boldsymbol{R}}$. Also,

$R^{*}(X) \cap R^{*}(X)=R^{*}(X) \in \boldsymbol{\tau}_{\boldsymbol{R}} ; R^{*}(X) \cap B_{R}(X)=B_{R}(X) \in \boldsymbol{\tau}_{\boldsymbol{R}}$ and $R^{*}(X) \cap B_{R}(X)=\varphi \in \boldsymbol{\tau}_{\boldsymbol{R}}$

Definition 2.2: Let $U$ be the universe, $R$ be an equivalence relation on $U$ and

$\boldsymbol{\tau}_{\boldsymbol{R}}=\left\{U, \varphi, R_{*}(X)\right\}$,

$\left.R^{*}(X), B_{R}(X)\right\}$ where $X \subseteq U . \boldsymbol{\tau}_{\boldsymbol{R}}$ satisfies the following axioms:

i) $\quad U$ and $\varphi \in \boldsymbol{\tau}_{\boldsymbol{R}}$

ii) The union of the elements of any subcollection of $\boldsymbol{\tau}_{R}$ is in $\boldsymbol{\tau}_{R}$.

iii) The intersection of the elements of any finite subcollection of $\boldsymbol{\tau}_{\boldsymbol{R}}$ is in $\boldsymbol{\tau}_{\boldsymbol{R}}$.

$\boldsymbol{\tau}_{\boldsymbol{R}}$ forms a topology on $U$ called as the nano topology on $U$ with respect to $X$. We call $\left(U, \boldsymbol{\tau}_{\boldsymbol{R}}, X\right)$ as the nano topological space.

Example 2.3: Let $U=\{a, b, c, d, e\}, U / R=\{\{a, b\},\{c, d\},\{e\}\}$, the family of equivalence classes of $U$ by the equivalence relation $R$ and $X=\{a, c, d\}$. Then $R^{*}(X)=\{a, b, c, d\}, R^{*}(X)=\{c, d\}$ and $B_{R}(X)=\{a, b\}$. Therefore the nano topology $\boldsymbol{\tau}_{\boldsymbol{R}}=\{U$ $\varphi,\{a, b, c, d\},\{c, d\},\{a, b\}\}$.

Proposition 3.4: If $\boldsymbol{\tau}_{\boldsymbol{R}}$ is the nano topology on $U$ with respect to $X$, then the set $B=\left\{U, R_{*}(X), B_{R}(X)\right\}$ is the basis for $\boldsymbol{\tau}_{\boldsymbol{R}}$. Proof:

i) $\bigcup_{A \in B} A=U$

ii) Consider $U$ and $R_{*}(X)$ from $B$. Let $W=R_{*}(X)$. Since $U \cap R_{*}(X)=R_{*}(X), W \subset U \cap R_{*}(X)$ and every $x$ in $U \cap R_{*}(X)$ belongs to $W$. If we consider $U$ and $B_{R}(X)$ from $B$, taking $W=B_{R}(X), W \subset U \cap B_{R}(X)$ and every $x$ in $U \cap B_{R}(X)$ belongs to $W$, since $U \cap B_{R}(X)=B_{R}(X)$. And when we consider $R_{*}(X)$ and $B_{R}(X), R_{*}(X) \cap B_{R}(X)=\varphi$. Thus, $B$ is a basis for $\boldsymbol{\tau}_{\boldsymbol{R}}$

Definition 2.4: Let $U$ be the universe and $R$ be an equivalence relation on $U$. Let $\boldsymbol{\tau}_{\boldsymbol{R}}$ be the nano topology on $U$ and $\beta_{R}$ be the basis for $\boldsymbol{\tau}_{\boldsymbol{R}}$. A subset $M$ of $A$, the set of attributes is called the core of $R$ if $\beta_{M} \neq \beta_{R-(r)}$ for every $r$ in $M$. That is, a core of $R$ is a subset of attributes which is such that none of its elements can be removed without affecting the classification power of attributes.

\section{3- Nano Topology in Power System Control Center}

The operation of a power system is intrinsically complex due to high degree of uncertainty and the large number of variables involved [5]. The various supervision and control actions require the presence of an operator, who must be capable of efficiently responding to the most diverse requests, by handling various types of data and information [6].

One of the most important operator task is to determine the current operational state of the system. To accomplish this task, the operator receives many data measured into the system. These data can be analogical (e.g., position of circuit breakers) or digital (e.g., real power flow in a specific transmission line). By handling these data, the operator tries to built an image of the operation point of the power system supervised for him/her.

The analysis performed by the operator tries to make a classification of the operational state of the system in one of the two states: normal, and restorative.

In the first state, normal state, all loads are supplied and all measurements are inside of the nominal rates. For the restorative operational state, some loads are not supplied, i.e., there was a loading shedding process .

The problem found by the operator is to use all available data in his/her analyses. A huge number of data and information in a control center database must be manipulated and, mainly, composed to allow the operator to have a visualization of the current state of the system. The manipulation of all data/information is not an easy task.

4- This paper presents an example of an alternative approach to help the operators to produce the classification of the system in its possible states. This approach is based on the Nano Topological Analysis, proposed by Lellis Thivagar et al [7]. 


\section{4- Example}

Let Table 1, where the information system proposed is composed by $R=\{A, B, C, D, R /\{A, B, C, D\} \Rightarrow\{R\}\}$, being $A, B, C, D$ and $R$, the attributes for transmission lines $A$ and $B$, self-generation, self-load, and operation point, respectively. Using these values. In many cases, power system problems can be analyzed by considering only an incomplete set of cases. In power system, it should be noticed that it is impossible to get all possible intervals of each measure and produce all combinations among them, because the number of observed points is enormous. Thus, the produced set of examples in a real system is always incomplete.

Table 1. The information system

\begin{tabular}{|c|c|c|c|c|c|}
\hline EX. & Trans.Line $A$ & Trans.Line $B$ & $\begin{array}{c}\text { Self-Gen } \\
\text { C }\end{array}$ & Self-Load $D$ & Result \\
\hline$E 1$ & medium & medium & Medium & medium & Normal \\
\hline E2 & medium & Iow & Low & medium & Rest \\
\hline E3 & medium & low & Low & medium & Normal \\
\hline$E 4$ & low & low & Low & high & Rest \\
\hline E5 & low & medium & Medium & medium & Rest \\
\hline E6 & medium & medium & Low & high & Normal \\
\hline$E 7$ & medium & medium & Low & Iow & Rest \\
\hline$E 8$ & medium & medium & Low & high & Normal \\
\hline
\end{tabular}

$$
U=\left\{E_{1}, E_{2}, E_{3}, E_{4}, E_{5}, E_{6}, E_{7}, E_{8}\right\}
$$

\section{Case 1}

$$
\text { Let } X=\left\{E_{1}, E_{3}, E_{6}, E_{8}\right\}
$$

Let $R$ be the equivalence relation on $U$ with respect to the condition attributes. The family of equivalence classes corresponding to $r$ is given by

$$
\boldsymbol{U} / \boldsymbol{I}(\boldsymbol{R})=\left\{\left\{\boldsymbol{E}_{1}\right\},\left\{\boldsymbol{E}_{2}, \boldsymbol{E}_{3}\right\},\left\{\boldsymbol{E}_{4}\right\},\left\{\boldsymbol{E}_{5}\right\},\left\{\boldsymbol{E}_{6}, \boldsymbol{E}_{\mathbf{8}}\right\},\left\{\boldsymbol{E}_{7}\right\}\right\} .
$$

The lower and upper approximations of $X$ with respect to $R$ are given by

$$
\boldsymbol{R}_{*}(X)=\left\{\boldsymbol{E}_{1}, \boldsymbol{E}_{6}, \boldsymbol{E}_{\mathbf{8}}\right\} \quad \boldsymbol{R}^{*}(\boldsymbol{X})=\left\{\boldsymbol{E}_{1}, \boldsymbol{E}_{2}, \boldsymbol{E}_{3}, \boldsymbol{E}_{\mathbf{6}}, \boldsymbol{E}_{\mathbf{8}}\right\}
$$

The nano topology on $U$ with respect to $X$ is given by

$\tau_{R}=\left\{U, \varphi,\left\{E_{1}, E_{6}, E_{8}\right\},\left\{E_{1}, E_{2}, E_{3}, E_{6}, E_{8}\right\},\left\{E_{2}, E_{3}\right\}\right\}$

The basis for this topology $\boldsymbol{\tau}_{\boldsymbol{R}}$ is given by $\boldsymbol{\beta}_{\boldsymbol{R}}=\left\{\boldsymbol{U},\left\{\boldsymbol{E}_{\mathbf{1}}, \boldsymbol{E}_{\mathbf{6}}, \boldsymbol{E}_{\mathbf{8}}\right\},\left\{\boldsymbol{E}_{\mathbf{2}}, \boldsymbol{E}_{\mathbf{3}}\right\}\right\}$

1- If we remove the attribute trans. Line A from the set of condition attributes, the family of equivalence classes corresponding to the resulting set of attributes is given by

$$
\boldsymbol{U} / \boldsymbol{I}(\boldsymbol{R}-(\boldsymbol{A}))=\left\{\left\{\boldsymbol{E}_{1}, \boldsymbol{E}_{5}\right\},\left\{\boldsymbol{E}_{2}, \boldsymbol{E}_{3}\right\},\left\{\boldsymbol{E}_{4}\right\},\left\{\boldsymbol{E}_{\mathbf{6}}, \boldsymbol{E}_{\mathbf{8}}\right\},\left\{\boldsymbol{E}_{7}\right\}\right\} \text {. }
$$

The corresponding lower and upper approximation are given by

$$
\begin{aligned}
& (\boldsymbol{R}-(\boldsymbol{A}))_{*}(\boldsymbol{X})=\left\{\boldsymbol{E}_{\mathbf{6}}, \boldsymbol{E}_{\mathbf{8}}\right\} ;(\boldsymbol{R}-(\boldsymbol{A}))^{*}(\boldsymbol{X})=\left\{\boldsymbol{E}_{\mathbf{1}}, \boldsymbol{E}_{\mathbf{2}}, \boldsymbol{E}_{\mathbf{3}}, \boldsymbol{E}_{\mathbf{5}}, \boldsymbol{E}_{\mathbf{6}}, \boldsymbol{E}_{\mathbf{8}}\right\} \\
& \text { And hence } \boldsymbol{\tau}_{\boldsymbol{R}-(\boldsymbol{A})}=\left\{\boldsymbol{U}, \boldsymbol{\varphi},\left\{\boldsymbol{E}_{\mathbf{6}}, \boldsymbol{E}_{\mathbf{8}}\right\},\left\{\boldsymbol{E}_{\mathbf{1}}, \boldsymbol{E}_{\mathbf{2}}, \boldsymbol{E}_{\mathbf{3}}, \boldsymbol{E}_{\mathbf{5}}, \boldsymbol{E}_{\mathbf{6}}, \boldsymbol{E}_{\mathbf{8}}\right\},\left\{\boldsymbol{E}_{\mathbf{1}}, \boldsymbol{E}_{\mathbf{2}}, \boldsymbol{E}_{\mathbf{3}}, \boldsymbol{E}_{\mathbf{5}}\right\}\right\}
\end{aligned}
$$

And its basis $\boldsymbol{\beta}_{\boldsymbol{R}-(\boldsymbol{A})}=\left\{\boldsymbol{U},\left\{\boldsymbol{E}_{6}, \boldsymbol{E}_{\mathbf{8}}\right\},\left\{\boldsymbol{E}_{1}, \boldsymbol{E}_{2}, \boldsymbol{E}_{3}, \boldsymbol{E}_{5}\right\}\right\} \neq \boldsymbol{\beta}_{R}$

2- If we remove the attribute trans. Line $B$ from the set of condition attributes, the family of equivalence classes corresponding to the resulting set of attributes is given by 
$\boldsymbol{U} / \boldsymbol{I}(\boldsymbol{R}-(\boldsymbol{B}))=\left\{\left\{\boldsymbol{E}_{1}\right\},\left\{\boldsymbol{E}_{2}, \boldsymbol{E}_{3}\right\},\left\{\boldsymbol{E}_{4}\right\},\left\{\boldsymbol{E}_{\mathbf{5}}\right\}\left\{\boldsymbol{E}_{\mathbf{6}}, \boldsymbol{E}_{\mathbf{8}}\right\},\left\{\boldsymbol{E}_{7}\right\}\right\}$

Which is the same as $\mathbf{U} / \mathbf{I}(\mathbf{R})$ and hence $\boldsymbol{\tau}_{\mathbf{R}-(\mathbf{B})}=\boldsymbol{\tau}_{\mathbf{R}}$ and $\boldsymbol{\beta}_{\mathbf{R}-(\mathbf{B})}=\boldsymbol{\beta}_{\mathbf{R}}$

3- If we remove the attribute Self Gen. C from the set of condition attributes, the family of equivalence classes corresponding to the resulting set of attributes is given by

$\mathbf{U} / \mathbf{I}(\mathbf{R}-(\mathbf{C}))=\left\{\left\{\mathbf{E}_{1}\right\},\left\{\mathbf{E}_{\mathbf{2}}, \mathbf{E}_{3}\right\},\left\{\mathbf{E}_{4}\right\},\left\{\mathbf{E}_{5}\right\}\left\{\mathbf{E}_{6}, \mathbf{E}_{\mathbf{8}}\right\},\left\{\mathbf{E}_{7}\right\}\right\}$

Which is the same as $\mathbf{U} / \mathbf{I}(\mathbf{R})$ and hence $\tau_{\mathbf{R}-(\mathbf{C})}=\tau_{\mathbf{R}}$ and $\boldsymbol{\beta}_{\mathbf{R}-(\mathbf{C})}=\boldsymbol{\beta}_{\mathbf{R}}$

4- If we remove the attribute Self Load $D$ from the set of condition attributes, the family of equivalence classes corresponding to the resulting set of attributes is given by

$\boldsymbol{U} / \boldsymbol{I}(\boldsymbol{R}-(\boldsymbol{D}))=\left\{\left\{\boldsymbol{E}_{1}\right\},\left\{\boldsymbol{E}_{2}, \boldsymbol{E}_{3}\right\},\left\{\boldsymbol{E}_{4}\right\},\left\{\boldsymbol{E}_{5}\right\},\left\{\boldsymbol{E}_{6}, \boldsymbol{E}_{7}, \boldsymbol{E}_{\mathbf{8}}\right\}\right\}$.

The corresponding lower and upper approximation are given by

$$
(\boldsymbol{R}-(\boldsymbol{D}))_{*}(\boldsymbol{X})=\left\{\boldsymbol{E}_{1}\right\} ;(\boldsymbol{R}-(\boldsymbol{D}))^{*}(\boldsymbol{X})=\left\{\boldsymbol{E}_{\mathbf{1}}, \boldsymbol{E}_{2}, \boldsymbol{E}_{3}, \boldsymbol{E}_{6}, \boldsymbol{E}_{7}, \boldsymbol{E}_{8}\right\}
$$

And hence $\quad \tau_{R-(D)}=\left\{U, \varphi,\left\{E_{1}\right\},\left\{E_{1}, E_{2}, E_{3}, E_{6}, E_{7}, E_{8}\right\},\left\{E_{2}, E_{3}, E_{6}, E_{7}, E_{8}\right\}\right\}$

And its basis $\boldsymbol{\beta}_{\boldsymbol{R}-(\boldsymbol{D})}=\left\{\boldsymbol{U},\left\{\boldsymbol{E}_{1}\right\},\left\{\boldsymbol{E}_{2}, \boldsymbol{E}_{3}, \boldsymbol{E}_{6}, \boldsymbol{E}_{7}, \boldsymbol{E}_{\mathbf{8}}\right\}\right\} \neq \boldsymbol{\beta}_{\boldsymbol{R}}$

If $\boldsymbol{M}=\{\boldsymbol{A}, \boldsymbol{D}\}$, Then the basis of thr rough topology corresponding to $\mathrm{M}$ is given by

$\boldsymbol{\beta}_{M}=\left\{U,\left\{E_{6}, E_{8}\right\},\left\{E_{1}, E_{2}, E_{3}\right\}\right\}$

Also $\boldsymbol{\beta}_{\boldsymbol{M}} \neq \boldsymbol{\beta}_{\boldsymbol{R}-(\boldsymbol{X})}$ for all $\mathrm{x}$ in M. Therefore, CORE $=\{A, D\}$

\section{Case 2}

$$
\text { Let } X=\left\{E_{2}, E_{4}, E_{5}, E_{7}\right\}
$$

Let $R$ be the equivalence relation on $U$ with respect to the condition attributes. The family of equivalence classes corresponding to $r$ is given by

$$
\boldsymbol{U} / \boldsymbol{I}(\boldsymbol{R})=\left\{\left\{\boldsymbol{E}_{1}\right\},\left\{\boldsymbol{E}_{2}, \boldsymbol{E}_{3}\right\},\left\{\boldsymbol{E}_{4}\right\},\left\{\boldsymbol{E}_{5}\right\},\left\{\boldsymbol{E}_{6}, \boldsymbol{E}_{\mathbf{8}}\right\},\left\{\boldsymbol{E}_{7}\right\}\right\} \text {. }
$$

The lower and upper approximations of $X$ with respect to $R$ are given by

$$
\boldsymbol{R}_{*}(\boldsymbol{X})=\left\{\boldsymbol{E}_{4}, \boldsymbol{E}_{5}, \boldsymbol{E}_{7}\right\} \quad \boldsymbol{R}^{*}(\boldsymbol{X})=\left\{\boldsymbol{E}_{2}, \boldsymbol{E}_{\mathbf{3}}, \boldsymbol{E}_{\mathbf{4}}, \boldsymbol{E}_{\mathbf{5}}, \boldsymbol{E}_{7}\right\}
$$

The rough topology on $U$ with respect to $X$ is given by

$$
\tau_{R}=\left\{U, \varphi,\left\{E_{4}, E_{5}, E_{7}\right\},\left\{E_{2}, E_{3}, E_{4}, E_{5}, E_{7}\right\},\left\{E_{2}, E_{3}\right\}\right\}
$$

The basis for this topology $\boldsymbol{\tau}_{\boldsymbol{R}}$ is given by $\boldsymbol{\beta}_{\boldsymbol{R}}=\left\{\boldsymbol{U},\left\{\boldsymbol{E}_{\mathbf{4}}, \boldsymbol{E}_{\mathbf{5}}, \boldsymbol{E}_{\mathbf{7}}\right\},\left\{\boldsymbol{E}_{\mathbf{2}}, \boldsymbol{E}_{3}\right\}\right\}$

1- If we remove the attribute trans. Line A from the set of condition attributes, the family of equivalence classes corresponding to the resulting set of attributes is given by

$$
\boldsymbol{U} / \boldsymbol{I}(\boldsymbol{R}-(\boldsymbol{A}))=\left\{\left\{\boldsymbol{E}_{1}, \boldsymbol{E}_{5}\right\},\left\{\boldsymbol{E}_{2}, \boldsymbol{E}_{3}\right\},\left\{\boldsymbol{E}_{4}\right\},\left\{\boldsymbol{E}_{6}, \boldsymbol{E}_{8}\right\},\left\{\boldsymbol{E}_{7}\right\}\right\} .
$$

The corresponding lower and upper approximation are given by

$(\boldsymbol{R}-(\boldsymbol{A}))_{*}(\boldsymbol{X})=\left\{\boldsymbol{E}_{4}, \boldsymbol{E}_{7}\right\} ;(\boldsymbol{R}-(\boldsymbol{A}))^{*}(\boldsymbol{X})=\left\{\boldsymbol{E}_{1}, \boldsymbol{E}_{2}, \boldsymbol{E}_{3}, \boldsymbol{E}_{4}, \boldsymbol{E}_{5}, \boldsymbol{E}_{7}\right\}$

And hence $\quad \tau_{R-(A)}=\left\{U, \varphi,\left\{E_{4}, E_{7}\right\},\left\{E_{1}, E_{2}, E_{3}, E_{4}, E_{5}, E_{7}\right\},\left\{E_{1}, E_{2}, E_{3}, E_{5}\right\}\right\}$

And its basis $\boldsymbol{\beta}_{R-(A)}=\left\{\boldsymbol{U},\left\{\boldsymbol{E}_{4}, \boldsymbol{E}_{7}\right\},\left\{\boldsymbol{E}_{1}, \boldsymbol{E}_{2}, \boldsymbol{E}_{3}, \boldsymbol{E}_{5}\right\}\right\} \neq \boldsymbol{\beta}_{\boldsymbol{R}}$

2- If we remove the attribute trans. Line $B$ from the set of condition attributes, the family of equivalence classes corresponding to the resulting set of attributes is given by

$$
\boldsymbol{U} / \boldsymbol{I}(\boldsymbol{R}-(\boldsymbol{B}))=\left\{\left\{\boldsymbol{E}_{1}\right\},\left\{\boldsymbol{E}_{2}, \boldsymbol{E}_{3}\right\},\left\{\boldsymbol{E}_{4}\right\},\left\{\boldsymbol{E}_{5}\right\}\left\{\boldsymbol{E}_{\mathbf{6}}, \boldsymbol{E}_{\mathbf{8}}\right\},\left\{\boldsymbol{E}_{7}\right\}\right\}
$$


Which is the same as $\boldsymbol{U} / \boldsymbol{I}(\boldsymbol{R})$ and hence $\boldsymbol{\beta}_{\boldsymbol{R}-(\boldsymbol{B})}=\boldsymbol{\beta}_{\boldsymbol{R}}$

3- If we remove the attribute Self Gen. $C$ from the set of condition attributes, the family of equivalence classes corresponding to the resulting set of attributes is given by

$\boldsymbol{U} / \boldsymbol{I}(\boldsymbol{R}-(\boldsymbol{C}))=\left\{\left\{\boldsymbol{E}_{1}\right\},\left\{\boldsymbol{E}_{2}, \boldsymbol{E}_{3}\right\},\left\{\boldsymbol{E}_{4}\right\},\left\{\boldsymbol{E}_{5}\right\}\left\{\boldsymbol{E}_{6}, \boldsymbol{E}_{\mathbf{8}}\right\},\left\{\boldsymbol{E}_{7}\right\}\right\}$

Which is the same as $U / I(R)$ and hence $\tau_{R-(C)}=\tau_{R}$ and $\beta_{R-(C)}=\beta_{R}$

1- If we remove the attribute Self Load $D$ from the set of condition attributes, the family of equivalence classes corresponding to the resulting set of attributes is given by

$$
\boldsymbol{U} / \boldsymbol{I}(\boldsymbol{R}-(\boldsymbol{D}))=\left\{\left\{\boldsymbol{E}_{1}\right\},\left\{\boldsymbol{E}_{2}, \boldsymbol{E}_{3}\right\},\left\{\boldsymbol{E}_{4}\right\},\left\{\boldsymbol{E}_{5}\right\},\left\{\boldsymbol{E}_{6}, \boldsymbol{E}_{7}, \boldsymbol{E}_{8}\right\}\right\} .
$$

The corresponding lower and upper approximation are given by

$$
(\boldsymbol{R}-(\boldsymbol{D}))_{*}(\boldsymbol{X})=\left\{\boldsymbol{E}_{4}, \boldsymbol{E}_{5}\right\} ;(\boldsymbol{R}-(\boldsymbol{D}))^{*}(\boldsymbol{X})\left\{\boldsymbol{E}_{2}, \boldsymbol{E}_{3}, \boldsymbol{E}_{4}, \boldsymbol{E}_{5}, \boldsymbol{E}_{6}, \boldsymbol{E}_{7}, \boldsymbol{E}_{\mathbf{8}}\right\}
$$

And hence

$$
\begin{aligned}
& \tau_{R-(D)}=\left\{U, \varphi,\left\{E_{4}, E_{5}\right\},\left\{E_{2}, E_{3}, E_{4}, E_{5}, E_{6}, E_{7}, E_{8}\right\},\left\{E_{2}, E_{3}, E_{6}, E_{7}, E_{8}\right\}\right\} \\
& \text { And its basis } \boldsymbol{\beta}_{\boldsymbol{R}-(\boldsymbol{D})}=\left\{\boldsymbol{U},\left\{\boldsymbol{E}_{\mathbf{4}}, \boldsymbol{E}_{5}\right\},\left\{\boldsymbol{E}_{2}, \boldsymbol{E}_{3}, \boldsymbol{E}_{\mathbf{6}}, \boldsymbol{E}_{7}, \boldsymbol{E}_{\mathbf{8}}\right\}\right\} \neq \boldsymbol{\beta}_{\boldsymbol{R}} \\
& \text { If } M=\{A, D\}, U / I(r)=\left\{\left\{E_{1}, E_{2}, E_{3}\right\},\left\{E_{4}, E_{5}\right\},\left\{E_{6}, E_{8}\right\},\left\{E_{7}\right\}\right\}, \\
& \boldsymbol{r}_{*}(\boldsymbol{X})=\left\{\boldsymbol{E}_{\mathbf{4}}, \boldsymbol{E}_{\mathbf{5}}, \boldsymbol{E}_{7}\right\} \\
& \boldsymbol{r}^{*}(\boldsymbol{X})=\left\{\boldsymbol{E}_{\mathbf{1}}, \boldsymbol{E}_{2}, \boldsymbol{E}_{3}, \boldsymbol{E}_{\mathbf{4}}, \boldsymbol{E}_{\mathbf{5}}, \boldsymbol{E}_{7}\right\}
\end{aligned}
$$

Where $r$ is the equivalence relation on $U$ with respect to $M$.Therefore ,

$$
\boldsymbol{\beta}_{\boldsymbol{M}}=\left\{\boldsymbol{U},\left\{\boldsymbol{E}_{4}, \boldsymbol{E}_{5}, \boldsymbol{E}_{7}\right\},\left\{\boldsymbol{E}_{\mathbf{1}}, \boldsymbol{E}_{2}, \boldsymbol{E}_{3}\right\}\right\} \neq \boldsymbol{\beta}_{\boldsymbol{R}-(\boldsymbol{X})} \text { for all x in M. Therefore, here again }
$$

CORE $=\{A, D\}$

\section{5- Observation of the two cases}

we conclude that Trans. Line A and Self Load D are the key attributes that can cover all possible intervals of each measure and produce all combinations among them in spite of the enormous number of observed points.

\section{Conclusion}

The suggested method for reduction and decision rule extraction is based on applying nano topology concepts which are suitable models for uncertain analysis. This will open up the way for obtaining quantitive measures from qualitative data without coding .

\section{REFERENCES}

1. Bisaria,j., Srivastava,N., Pardasani,K. A Rough Set Model for Sequential Pattern Mining with Constraints, (IJCNS) International Journal of Computer and Network Security, vol. 1, No. 2, Nov. 2009.

2. Chuangyin ,Y., Jiye L. and Dawei T, Set- valued information systems, Information Sciences 179 , 2809-2832,2009.

3. Chouchoulas,A., Shen,Q., Rough set-aided keyword reduction for text categorisation. Applied Artificial Intelligence, vol. 15, pp. 843-873,2001.

4. Duo C., Du,W., Chao.X., Zhu-Rong Wang, A Rough Set-Based Hierarchical Clustering Algorithm for Categorical Data, International Journal of Information Technology, vol. 12, No.3, 2006.

5. Germano,L.Application of Rough Sets in Power System Control Center Data Mining, IEEE,2002.

6. Meng,Z. and Zhongzhi S., A fast approach to attribute reduction in incomplete decision systems with tolerance re-lation - based rough sets, Information Sciences, 179, 2774-2793,2009.

7. Pawlak,Z. Rough set theory and its applications, Telecommunications and Information .

8. Technology 3,7-10,2002.

9. Pawlak,Z., Rough sets, J. Computational Information Sciences, vol.11, 1982, pp.341-345. 
10. Pawlak,Z., Classification of Objects by Means of Attributes, Reports, vol. 429, 1981, Institute of Computer Science, Polish Academy of Sciences, Warsaw, Poland.

11. Pawlak,Z., Rough Relations, Reports, vol. 435, 1981, Institute of Computer Science, Polish Academy of Sciences, Warsaw, Poland

12. Pawlak,Z., Information systems-theoretical foundations, Information Systems $6,205-218,1981$.

13. Pawlak,Z., Rough sets, J. Computational Information Sciences, vol.11, pp.341-345,1982.

14. Rady,E., Kozae,A., and Abd El-Monsef,M., 2004, Gene-ralized rough sets, Chaos, Solitons and Fractals 21, 4953.

15. Sai,Y., Nie,P., Xu, R., Huang,J., A Rough Set Approach to Mining Concise Rules from Inconsistent Data, IEEE International Conference on Granular Computing, pp. 333-336, 10-12 May 2006.

16. Thivagar,L., Richard,M.,On Nano forms of weakly open sets, International Journal of Mathematical and Statistics Invention, 1(1):31-37,2012.

17. Valiquette,B., Germano, L., and Mukhedkar,D.Vol.6, No.3, pp. 1315-1322 An Expert System Based Diagnosis and Advisor Tool for Teaching Power System Operation Emergency Control Strategies, IEEE Trans. On Power Systems, Vol.6, No.3 (August 1991) pp. 1315-1322,1991. 